\title{
BMJ Open Pregnancy outcomes among Chinese women with and without systemic lupus erythematosus: a retrospective cohort study
}

Jiayue Wu, ${ }^{1,2,3}$ Jinghang Ma, ${ }^{1,2}$ Chunde Bao, ${ }^{4,5}$ Wen Di, ${ }^{1,2}$ Wei-Hong Zhang ${ }^{3,6}$

To cite: Wu J, Ma J, Bao C, et al. Pregnancy outcomes among Chinese women with and without systemic lupus erythematosus: a retrospective cohort study. BMJ Open 2018:8:e020909. doi:10.1136/ bmjopen-2017-020909

- Prepublication history for this paper is available online. To view these files, please visit the journal online (http://dx.doi org/10.1136/bmjopen-2017020909).

WD and W-HZ contributed equally.

Received 1 December 2017 Revised 13 February 2018 Accepted 15 March 2018

\section{Check for updates}

${ }^{1}$ Department of Obstetrics and Gynecology, School of Medicine Renji Hospital, Shanghai Jiao Tong University, Shanghai, China ${ }^{2}$ Shanghai Key Laboratory of Gynecologic Oncology, Shanghai, China

${ }^{3}$ International Centre for Reproductive Health (ICRH), Ghent University, Gent, Belgium ${ }^{4}$ Department of Rheumatology, School of Medicine, Renji Hospital, Shanghai Jiao Tong University, Shanghai, China ${ }^{5}$ Shanghai Institute of Rheumatology, Shanghai, China ${ }^{6}$ ResearchLaboratory for Human Reproduction, Facultyof Medicine, Université Libre de Bruxelles (ULB), Brussels, Belgium

Correspondence to DrWen Di; diwen163@163.com and Prof. Wei-Hong Zhang; WeiHong.Zhang@UGent.be

\section{ABSTRACT}

Objective To completely and quantifiably determine the effect of systemic lupus erythematosus (SLE) on pregnancy outcomes in a Chinese cohort.

Design A retrospective cohort study.

Setting Data were collected at a tertiary medical centre located in Shanghai, China, from September 2011 to May 2017.

Participants We assigned 338 pregnant women with SLE to the study cohort and 1014 randomly selected pregnant women without SLE (three for every woman with SLE) to a comparison cohort. The relevant medical records of all pregnant women were retrospectively reviewed. Cases of multiple pregnancy and cases in which an artificial abortion was performed for personal reasons were excluded.

Primary and secondary outcome measures Maternal and fetal outcomes were primary outcomes, and management of antenatal care was the secondary outcome.

Results The risks of pregnancy-induced hypertension (OR 2.68, 95\% Cl 1.75 to 4.09), pre-eclampsia (OR $3.13,95 \% \mathrm{Cl} 1.95$ to 5.03 ) and premature rupture of membranes (OR $2.53,95 \% \mathrm{Cl} 1.46$ to 4.40 ) were significantly different between women with and without SLE. Gestational diabetes was negatively associated with SLE in pregnant women (OR $0.49,95 \% \mathrm{Cl} 0.28$ to 0.85 ). Pregnant women with SLE displayed significantly higher rates of fetal loss (OR 10.23, 95\% Cl 5.08 to 20.59), including spontaneous abortion (OR 4.42, 95\% Cl 1.52 to 12.80), therapeutic abortion (OR 16.57, 95\% Cl 5.80 to 47.35) and stillbirth (OR 13.25, 95\% Cl 1.49 to 118.11 ), and a higher risk of preterm birth (OR 3.15, 95\% Cl 2.21 to 4.50 ), intrauterine growth restriction (OR $2.20,95 \% \mathrm{Cl}$ 1.35 to 3.58 ), a child who was small for the gestational age (OR 1.86, 95\% Cl 1.11 to 3.13 ), a caesarean section (OR $4.73,95 \% \mathrm{Cl} 3.30$ to 6.80 ) or a neonatal intensive care unit admission (OR 3.48, 95\% $\mathrm{Cl} 2.21$ to 5.48 ) than women in the non-SLE population after adjusting for confounding factors.

Conclusions In this study, SLE significantly increased the risk of adverse pregnancy outcomes. Therefore, a preconception assessment and close antenatal monitoring by both rheumatologists and obstetricians should be performed in pregnant women with SLE.

\section{Strengths and limitations of this study}

- This study represents the first comparison of maternal and fetal outcomes between pregnant women with and without systemic lupus erythematosus (SLE) in a Chinese cohort.

- Additionally, the present study included large samples of both patients with SLE and controls comprising a total of 1350 individuals, and no data were missing for any of these patients.

- Furthermore, the maternal and fetal outcomes evaluated in this study were comprehensive and reflect almost every key aspect of pregnancy.

- One limitation of this retrospective study was inherent biases, including selection bias and information bias.

- The second limitation was the lack of some details regarding baseline population characteristics, for example, education, body mass index and family income, in the clinical records for some of the included participants.

\section{INTRODUCTION}

Systemic lupus erythematosus (SLE) is an autoimmune disease that involves multiple organs and most commonly occurs in young women, in whom it has an incidence of $0.3-31.5 / 100000$ person-years and prevalence of 3.2-517.5/100 $000 .^{1{ }^{2}}$ Women with SLE have a normal fertility rate, ${ }^{2}$ and pregnancy is therefore a frequent subject of interest for these patients. As treatments for pregnant women with SLE have improved and increasingly involve multidisciplinary management, pregnancy outcomes have dramatically improved in recent decades. ${ }^{3}$ Although several studies have compared pregnancy outcomes between women with SLE and the general population ${ }^{4-7}$ and reported that SLE is associated with adverse obstetric outcomes, few studies have examined these outcomes in a Chinese cohort. Therefore, we have compared maternal and fetal outcomes between women with SLE and women in the 
general population to completely and quantifiably clarify the effect of SLE on pregnancy outcomes in a Chinese cohort.

\section{MATERIALS AND METHODS Study sample}

This retrospective cohort study was performed in a single centre. Our study cohort consisted of pregnant patients with SLE who were treated in Ren Ji Hospital, Shanghai Jiao Tong University School of Medicine between September 2011 and May 2017. Multiple gestations and elective terminations for personal reasons were excluded. SLE was diagnosed according to the revised criteria for the classification of SLE developed by the American College of Rheumatology. ${ }^{8}$ All participants with SLE were evaluated by an experienced gynaecologist at least monthly and by a rheumatologist at least once per trimester. At each evaluation, a physical examination and laboratory tests were performed, and medications were adjusted as needed.

Our comparison cohort was randomly selected from women with singleton pregnancies who were not diagnosed with SLE and attended the same hospital. Control participants were matched with the study group by delivery time (three controls were selected for every woman with SLE) and received regular, routine antenatal care.

\section{Variables of interest}

Medical records from both cases and controls were retrospectively reviewed, and pregnancy outcomes were systematically evaluated.

\section{Population characteristics}

The baseline characteristics of the population were recorded to identify potential confounding factors. These factors included maternal age at delivery, region, nulliparity, history of spontaneous abortion and comorbidities. Region was categorised as city and rural areas. A history of spontaneous abortion was categorised as 0,1 and $\geq 2$, according to the numbers of miscarriages. Comorbidities included a prepregnancy diagnosis of hypertension or diabetes. None of the women in the study population had ever smoked or used alcohol during pregnancy; therefore, these two variables were not included in the study. For the SLE cohort, the history of SLE, pregestational SLE status, SLE clinical manifestations (including nephritis, mucocutaneous, haematological disorder, neurological disorder, arthritis, serositis and antiphospholipid syndrome (APS)), the laboratory test data and medications were also extracted. Laboratory data included a complete blood count, urinalysis and levels of serum albumin, 24-hour urinary protein, complement $3\left(\mathrm{C}_{3}\right)$, complement $4\left(\mathrm{C}_{4}\right)$, antinuclear antibodies, anti-double standard DNA antibodies (anti-dsDNA), anti-Smith antibodies (anti-Sm), anti-SSA/Ro antibodies, anti-SSB/La antibodies and antiphospholipid (aPL) antibodies, which included anticardiolipin antibodies, anti- $\beta 2$-glycoprotein
I antibodies and lupus anticoagulant. APS was defined according to the Sapporo criteria. ${ }^{9}$ All laboratory tests were performed using standardised methods.

The pregestational clinical status of patients with SLE was categorised into the remission stage, which was defined as a patient who took a low dose of or stopped prednisone treatment without clinical manifestations of SLE activity for more than 6 months prior to conception. The active stage was defined as patients presenting clinical manifestations of SLE activity, including central nervous system and renal involvement, vasculitis, arthritis, myositis, fever, rash, pleurisy, pericarditis and hypocomplementaemia. The initial onset during pregnancy was defined as a new onset of SLE during pregnancy.

\section{Maternal outcomes}

All outcome variables were dichotomous. The abstracted maternal outcomes and definitions are listed below.

- Pregnancy-induced hypertension (PIH): a de novo increase in blood pressure to $\geq 140 / 90 \mathrm{~mm} \mathrm{Hg}$ on at least two occasions $\geq 6$ hours apart observed after the 20th week of pregnancy.

- Pre-eclampsia: PIH with proteinuria $>0.3 \mathrm{~g} / \mathrm{L} /$ day in the absence of a urinary tract infection or the abrupt onset of hypertension and proteinuria after 20 weeks of gestation. Seizures were required for a diagnosis of eclampsia.

- HELLP (haemolysis, elevated liver enzymes and low platelet count) syndrome: the presence of haemolysis, high levels of lactate dehydrogenase or total bilirubin $>12 \mathrm{mg} / \mathrm{L}$, alanine aminotransferase levels more than twofold the upper normal value and thrombocytopenia $<100 \times 10^{9}$ cells $/ \mathrm{L}$.

- Gestational diabetes mellitus (GDM) was defined as any degree of glucose intolerance with onset or first recognition during pregnancy. ${ }^{10}$

- Premature rupture of membranes (PROM): a rupture of the amniotic sac prior to the onset of labour. We further divided this condition into term PROM and preterm PROM (PPROM), according to the neonatal gestational age.

- Postpartum haemorrhage (PPH) was defined as blood loss of $500 \mathrm{~mL}$ or more within 24 hours after birth. ${ }^{11}$

- Peripartum infection was defined as an infection of the genital tract occurring at any time between the onset of membrane rupture or labour and the 42nd postpartum day. ${ }^{12}$

- Maternal death: the death of a woman during pregnancy or within 42 days of the termination of a pregnancy, regardless of the duration or site of the pregnancy, of any cause related to or aggravated by the pregnancy or its management, but not any accidental or incidental causes. ${ }^{13}$

- An SLE flare was defined as a measurable increase in disease activity in one or more organ systems involving new or worsening clinical signs and symptoms and/or laboratory measurements that met the SLE diagnostic criteria ACR1997. ${ }^{8}$ The flare must 
be considered clinically significant by the assessor and a change or an increase in treatment is typically considered. $^{14}$

\section{Fetal outcomes}

All outcome variables were dichotomous and continuous. The abstracted fetal outcomes and definitions are listed below.

- Live birth: the birth of a living child.

- Fetal loss: defined as all pregnancies that did not end with a live birth, ${ }^{15}$ including spontaneous abortions, therapeutic abortions, stillbirths or intrauterine fetal deaths. Additional definitions were:

- Spontaneous abortion: spontaneous termination of a pregnancy prior to 28 weeks of gestation. ${ }^{16} \mathrm{We}$ further divided spontaneous abortions into $<10$ weeks and $\geq 10$ weeks.

- Therapeutic abortion: abortion for therapeutic reasons because the pregnancy might be a threat to maternal health, such as a life-threatening SLE flare or other severe obstetric complications, including severe thrombocytopenia or HELLP syndrome, etc or fetal lethal malformations, including the maldevelopment of cardiac anatomy.

- Stillbirth or intrauterine fetal death: any baby born without signs of life at $\geq 28$ completed weeks of gestation. $^{17}$

- Intrauterine growth restriction (IUGR): factors that limit the potential for the intrauterine growth of the fetus. ${ }^{18}$

- Small for gestational age (SGA): infants whose weight was lower than the lower $10 \%$ limit of the CI of the normal curve for gestational weight. ${ }^{19}$

- Neonatal death: live birth who died during the first 28 completed days of life. ${ }^{20}$

- Preterm birth: was defined as delivery <37 weeks of gestation. We further divided this condition into very preterm (28-32 weeks) and moderate to late preterm ( $\geq 32$ weeks), according to WHO definitions. $^{21}$

- Method of childbirth: caesarean section or vaginal birth. We further divided caesarean section into elective and emergency caesarean section, according to the indications for caesarean section. ${ }^{22}$

- Congenital malformation: all types of congenital malformation.

- Neonatal gender: male and female.

- Neonatal intensive care unit (NICU): neonates who required intensive medical attention and were admitted into a special area of the hospital called the NICU.

- 1 min and 5 min Apgar scores: an Apgar score $>7$ was defined as normal, whereas a score $<7$ was considered to indicate moderate or severe hypoxia. ${ }^{23}$

- Birth weight: neonatal birth weight is presented in grams.

- Gestational days: neonatal gestational days is presented in days.

\section{Statistical analysis}

Descriptive data are presented as means $₫$ SD or as frequencies. Categorical variables were analysed using the $\chi^{2}$ test or Fisher's exact probability test, as appropriate. Continuous variables were analysed using Student's t-test.

The denominator used in the analyses of all maternal outcomes, stillbirths and live births was all reported pregnancies. For all other fetal outcomes (excluding stillbirths and live births), the denominator was all live births.

Logistic regression analyses were performed to compute crude ORs with 95\% CIs for maternal and fetal outcomes. Logistic regression models were adjusted for potential confounding factors, including maternal age, region, nulliparity, a history of spontaneous abortion and comorbidities. These analyses yielded new adjusted ORs with 95\% CIs. All tests were two tailed, and $\mathrm{p}<0.05$ was considered statistically significant. All analyses were performed using SPSS V.22.0.

This study used a retrospective, observational design, the Medical Ethical Committee granted a waiver for informed consent for this study. Approval to obtain clinical data from the database was received from the Office of the Medical Director of the hospital. All patient information remained confidential.

\section{RESULTS}

\section{Population characteristics}

Three hundred and thirty-eight pregnant women with SLE (cases) and 1014 pregnant without SLE (controls) who were monitored between September 2011 and May 2017 were included. The distributions of the baseline characteristics of the population are shown in table 1 . Mean ages were not significantly different between the two cohorts. Women with SLE were more likely to reside in a city than the controls, and most of the pregnancies in women with SLE were nulliparous. A history of spontaneous abortion, particularly $\geq 2$ abortions, was more common in women with SLE. Significant differences in comorbid conditions were not observed between the women with SLE and the controls. The proportion of women in whom diabetes and/or hypertension was diagnosed prior to pregnancy was not significantly different between the SLE and non-SLE populations. Data for some variables, such as laboratory test data and SLE clinical manifestations, were not available for the non-SLE group because we do not perform these laboratory tests on patients without SLE manifestations. Regarding the SLE group, the mean duration of SLE was 5.6 \pm 4.3 years (range 0-20 years). Notably, $86.7 \%$ of pregnant women with SLE were in the remission stage, $1.8 \%$ were in the active age and $11.5 \%$ of women were first diagnosed with SLE during pregnancy.

The most common SLE clinical manifestations were mucocutaneous lesions, which were identified in $31.4 \%$ of patients, followed by lupus nephritis in $28.7 \%$, arthritis in $20.7 \%$, haematological disorders in $19.5 \%$, serositis in $5.0 \%$ and neurological disorders in $1.5 \%$. APS was 
Table 1 Baseline clinical characteristics of pregnant women with and without SLE

\begin{tabular}{|c|c|c|c|}
\hline Variables & SLE cases $(n=338)$ & Non-SLE cases $(n=1014)$ & $P$ values \\
\hline \multicolumn{4}{|l|}{ Characteristics } \\
\hline Age (years, mean $\pm S D$ ) & $29.5 \pm 4.0$ & $29.7 \pm 4.3$ & 0.59 \\
\hline \multicolumn{4}{|l|}{ History of spontaneous abortion (frequency) } \\
\hline 0 & $265(78.4 \%)$ & $903(89.1 \%)$ & $0.00^{\star \star}$ \\
\hline 1 & $46(13.6 \%)$ & $64(6.3 \%)$ & \\
\hline$\geq 2$ & $27(8.0 \%)$ & $47(4.6 \%)$ & \\
\hline History of SLE (years, mean $\pm S D$, range) & $5.6 \pm 4.3(0-20)$ & NA & \\
\hline \multicolumn{4}{|l|}{ Region } \\
\hline City & $237(70.1 \%)$ & $318(31.4 \%)$ & $0.00^{* *}$ \\
\hline Rural & $101(29.9 \%)$ & $695(68.6 \%)$ & \\
\hline Nulliparity & $291(86.1 \%)$ & $706(69.6 \%)$ & $0.00^{\star *}$ \\
\hline \multicolumn{4}{|l|}{ Clinical comorbidities } \\
\hline Prepregnancy diabetes & $1(0.3 \%)$ & $4(0.4 \%)$ & 1.00 \\
\hline Prepregnancy hypertension & $10(3.0 \%)$ & $21(2.1 \%)$ & 0.35 \\
\hline \multicolumn{4}{|l|}{ Pregestational SLE status } \\
\hline Remission stage & $293(86.7 \%)$ & NA & \\
\hline Active stage & $6(1.8 \%)$ & NA & \\
\hline Initial onset & $39(11.5 \%)$ & NA & \\
\hline \multicolumn{4}{|l|}{ SLE clinical manifestation } \\
\hline Nephritis & $97(28.7 \%)$ & NA & \\
\hline Mucocutaneous & $106(31.4 \%)$ & NA & \\
\hline Haematological disorder & $66(19.5 \%)$ & NA & \\
\hline Neurological disorder & $5(1.5 \%)$ & NA & \\
\hline Arthritis & $70(20.7 \%)$ & NA & \\
\hline Serositis & $17(5.0 \%)$ & NA & \\
\hline APS & $32(9.5 \%)$ & NA & \\
\hline \multicolumn{4}{|l|}{ Laboratory test during pregnancy } \\
\hline 24-Hour urinary protein level ( $g$, mean $\pm S D$, range) & $1.0 \pm 2.4(0.0-16.7)$ & NA & \\
\hline Anti-dsDNA & $261(77.2 \%)$ & NA & \\
\hline Anti-Ro/SSA & $150(44.4 \%)$ & NA & \\
\hline Anti-La/SSB & $47(13.9 \%)$ & NA & \\
\hline Anti-Sm & $20(5.9 \%)$ & NA & \\
\hline aPL & $46(13.6 \%)$ & NA & \\
\hline Hypocomplementania- $\mathrm{C}_{3}$ & $90(26.6 \%)$ & NA & \\
\hline Hypocomplementania- $\mathrm{C}_{4}$ & $60(17.8)$ & NA & \\
\hline
\end{tabular}

${ }^{\star *} \mathrm{P}<0.01$.

Anti-Sm, anti-Smith; Anti-dsDNA, anti-double stranded DNA; aPL, antiphospholipid; APS, antiphospholipid syndrome; $\mathrm{C}_{3}$, complement 3; $\mathrm{C}_{4}$, complement 3; NA, not applicable.

observed in $9.5 \%$ of the pregnant women with SLE. The mean 24-hour urinary protein level was $1.04 \pm 2.43 \mathrm{~g}$ (range, 0.01-16.7 g). Pregnant women were positive for autoantibodies at the following frequencies: anti-dsDNA in 261 patients (77.2\%), anti-Ro/SSA in 150 patients (44.4\%), anti-La/SSB in 47 patients (13.9\%) and anti-Sm in 20 patients $(5.9 \%)$. Forty-six patients were positive for aPL antibodies. $\mathrm{C}_{3}$ and $\mathrm{C}_{4}$ hypocomplementaemia was present in $90(26.6 \%)$ and 60 patients $(17.8 \%)$, respectively (table 1 ).

\section{Maternal outcomes}

The rate of adverse maternal outcomes was several fold higher in women with SLE than in the non-SLE population during pregnancy. PIH and pre-eclampsia/eclampsia were diagnosed in $19.2 \%$ and $14.2 \%$, respectively, of the 
Table 2 Maternal outcomes in patients with SLE and non-SLE

\begin{tabular}{|c|c|c|c|c|}
\hline Maternal outcomes & SLE $(n=338)$ & Non-SLE $(n=1014)$ & Crude OR $(95 \% \mathrm{Cl})$ & Adjusted OR†(95\% Cl) \\
\hline $\mathrm{PIH}(\%)$ & $65(19.2 \%)$ & $91(9.0 \%)$ & $2.42(1.71 \text { to } 3.41)^{\star \star}$ & $2.68(1.75 \text { to } 4.09)^{\star \star}$ \\
\hline Pre-eclampsia/eclampsia (\%) & $48(14.2 \%)$ & $59(5.8 \%)$ & $2.68(1.81 \text { to } 4.10)^{\star \star}$ & $3.13(1.95 \text { to } 5.03)^{\star \star}$ \\
\hline GDM (\%) & $19(5.6 \%)$ & $117(11.5 \%)$ & $0.46(0.28 \text { to } 0.75)^{\star *}$ & $0.49(0.28 \text { to } 0.85)^{\star}$ \\
\hline PROM (\%) & $67(19.8 \%)$ & $157(15.5 \%)$ & 1.35 (0.98 to 1.85$)$ & 1.35 (0.95 to 1.92$)$ \\
\hline PPROM (\%) & $29(8.5 \%)$ & $40(3.9 \%)$ & $2.29(1.39 \text { to } 3.75)^{\star *}$ & $2.53(1.46 \text { to } 4.40)^{\star *}$ \\
\hline PPH (\%) & $8(2.4 \%)$ & $18(1.8 \%)$ & $1.34(0.58$ to 3.11$)$ & 1.39 (0.54 to 3.53$)$ \\
\hline SLE flare (\%) & $81(24.0 \%)$ & NA & NA & NA \\
\hline
\end{tabular}

${ }^{*} \mathrm{P}<0.05$.

${ }^{\star *} \mathrm{P}<0.01$.

†Adjusted ORs were calculated using a logistic regression analysis and were adjusted for maternal age, region, nulliparity, history of spontaneous abortion, history of diabetes and history of hypertension.

GDM, gestational diabetes; HELLP, haemolysis, elevated liver enzymes and low platelet count; NA, not applicable; PPH, postpartum haemorrhage; PPROM, preterm premature rupture of membranes; PIH, pregnancy-induced hypertension; SLE, systemic lupus erythematosus; TPROM, term premature rupture of membranes.

women with SLE. After adjusting for confounding factors, the risks of PIH (OR 2.68, 95\% CI 1.75 to 4.09 ) or pre-eclampsia (OR 3.13, 95\% CI 1.95 to 5.03) remained high. The proportion of infection was low, and peripartum infections occurred in $0.9 \%(3 / 338)$ of the women with SLE and none of the control women. Interestingly, the rate of GDM was lower in women with SLE than in the controls, and the OR remained unchanged after adjusting for confounding factors (OR 0.49, 95\% CI 0.28 to 0.85$)$. Although we did not observe a difference in PROM between the two groups, a significant difference in PPROM was observed, and the OR remained high, even after adjusting for confounding factors (OR 2.53, 95\% CI 1.46 to 4.40$)$. Significant differences in other maternal outcomes, including PPH and HELLP syndrome, were not observed between the two groups. The SLE flare rate was $24.0 \%$ in the SLE group. Maternal deaths did not occur in either group (table 2 ).

\section{Fetal outcomes}

Women with SLE had a higher total fetal loss rate (OR 10.23, 95\% CI 5.08 to 20.59) than the non-SLE population after adjusting for confounding factors. Among the causes of fetal loss in women with SLE, 3.3\% experienced a spontaneous abortion, whereas $6.2 \%$ underwent a therapeutic abortion and $1.8 \%$ had a stillbirth. Furthermore, most of the spontaneous abortions occurred at $\geq 10$ weeks, regardless of the group. After adjusting for confounding factors, the risks of a spontaneous abortion (OR 4.42, 95\% CI 1.52 to 12.80 ), therapeutic abortion (OR 16.57, 95\% CI 5.80 to 47.35 ) or a stillbirth (OR 13.25, 95\% CI 1.49 to 118.11 ) were still high.

Among women who achieved a live birth, the rates of preterm were 3.15-fold higher in the pregnant women with SLE than in the controls. Both the very preterm and moderate to late preterm subgroups had higher adjusted ORs of 3.57 (95\% CI 1.65 to 7.72) and 2.76 (95\% CI 1.90 to 4.03), respectively. Caesarean births occurred at a 4.73fold higher rate in the SLE group, and $77 \%$ of patients with SLE tended to undergo an elective caesarean section. The rate of NICU admission for neonates was also higher in women with SLE. IUGR and SGA were observed in $12.3 \%$ and $10.0 \%$ of pregnancies in women with SLE, respectively. The adjusted ORs were 2.20 and 1.86, respectively, for each outcome (all $p$ values $<0.05$ ). Furthermore, we also observed lower neonatal birth weights $(2763.7 \pm 588.7 \mathrm{~g})$ and gestational days $(259.3 \pm 16.2$ days $)$ for pregnant women with SLE (both $\mathrm{p}<0.01$ ). No neonatal deaths occurred in either group. Finally, significant differences in the rates of congenital malformations, neonatal genders or the proportions of low Apgar scores were not observed between the cohorts (table 3). Only two cases of neonatal lupus occurred in the women with SLE, both of which presented as mucocutaneous lesions.

\section{Medications used during pregnancy by women with SLE}

Medications were recorded for the SLE group and mainly included five kinds of medicines. Of the 338 pregnant women with SLE, most $(97.6 \%)$ were treated with glucocorticoids, with a mean dose of $7.5 \mathrm{mg} /$ day (range $2.5-40 \mathrm{mg}$ ). Approximately, $75.4 \%$ and $74.6 \%$ of patients with SLE took hydroxychloroquine and low-dose aspirin (25-75 mg), respectively. Only a small proportion of patients $(3.8 \%)$ took azathioprine, and $22.2 \%$ of the patients used low-molecular-weight-heparin (LMWH) during their pregnancy.

\section{DISCUSSION}

\section{Principal findings}

In this study, pregnant women with SLE have a higher risk of adverse maternal outcomes, such as PIH, pre-eclampsia/eclampsia, PPROM and peripartum infection, than pregnant women without SLE. Furthermore, SLE 


\begin{tabular}{|c|c|c|c|c|}
\hline Fetal outcomes & SLE $(n=338)$ & Non-SLE $(n=1014)$ & Crude OR $(95 \% \mathrm{Cl})$ & Adjusted OR† $(95 \% \mathrm{Cl})$ \\
\hline Fetal loss (\%) & $38(11.2 \%)$ & $13(1.3 \%)$ & $9.75(5.13 \text { to } 18.55)^{\star \star}$ & $10.23(5.08 \text { to } 20.59)^{\star \star}$ \\
\hline Spontaneous abortion (\%) & $11(3.3 \%)$ & $7(0.7 \%)$ & $4.84(1.86 \text { to } 12.59)^{\star \star}$ & $4.42(1.52 \text { to } 12.80)^{\star \star}$ \\
\hline$<10$ weeks $(\%)$ & $2(0.6 \%)$ & $0(0.0 \%)$ & NA & NA \\
\hline$\geq 10$ weeks (\%) & $9(2.7 \%)$ & $7(0.7 \%)$ & $3.94(1.50 \text { to } 10.65)^{\star \star}$ & $3.79(1.25 \text { to } 11.44)^{\star}$ \\
\hline Therapeutic abortion (\%) & $21(6.2 \%)$ & $5(0.5 \%)$ & $13.37(5.00 \text { to } 35.74)^{\star \star}$ & $16.57(5.80 \text { to } 47.35)^{\star *}$ \\
\hline Stillbirth (\%) & $6(1.8 \%)$ & $1(0.1 \%)$ & $18.31(2.20 \text { to } 152.62)^{\star *}$ & $13.25(1.49 \text { to } 118.11)^{*}$ \\
\hline Live birth & SLE $(n=300)$ & Non-SLE $(n=1001)$ & & \\
\hline IUGR (\%) & $37(12.3 \%)$ & $57(5.7 \%)$ & $2.33(1.51 \text { to } 3.60)^{\star \star}$ & $2.20(1.35 \text { to } 3.58)^{\star \star}$ \\
\hline SGA (\%) & $30(10.0 \%)$ & $53(5.3 \%)$ & $1.99(1.25 \text { to } 3.17)^{\star *}$ & $1.86(1.11 \text { to } 3.13)^{\star}$ \\
\hline Preterm birth (\%) & $86(28.7 \%)$ & $133(13.3 \%)$ & $2.62(1.92 \text { to } 3.58)^{\star *}$ & $3.15(2.21 \text { to } 4.50)^{\star *}$ \\
\hline Very preterm (\%) & $15(5.0 \%)$ & $19(1.9 \%)$ & $2.72(1.37 \text { to } 5.42)^{\star \star}$ & $3.57(1.65 \text { to } 7.72)^{\star \star}$ \\
\hline Moderate to late preterm (\%) & $71(23.7 \%)$ & $114(11.4 \%)$ & $2.41(1.73 \text { to } 3.36)^{\star *}$ & $2.76(1.90 \text { to } 4.03)^{\star \star}$ \\
\hline Caesarean section (\%) & $255(85.0 \%)$ & $557(55.6 \%)$ & $4.51(3.21 \text { to } 6.35)^{\star \star}$ & $4.73(3.30 \text { to } 6.80)^{\star \star}$ \\
\hline Elective caesarean (\%) & $231(77.0 \%)$ & $298(29.7 \%)$ & $8.37(5.32 \text { to } 13.15)^{\star \star}$ & $9.27(5.65 \text { to } 15.21)^{* *}$ \\
\hline Emergency caesarean (\%) & $24(8.0 \%)$ & $259(25.8 \%)$ & $0.12(0.08 \text { to } 0.19)^{\star \star}$ & $0.11(0.07 \text { to } 0.18)^{\star \star}$ \\
\hline Congenital malformation (\%) & $4(1.3 \%)$ & $10(1.0 \%)$ & $1.34(0.42$ to 4.30$)$ & $1.60(0.43$ to 5.97$)$ \\
\hline Neonatal gender (boys) (\%) & $149(49.7 \%)$ & $548(54.7 \%)$ & $0.82(0.63$ to 1.06$)$ & 0.84 (0.63 to 1.11$)$ \\
\hline NICU (\%) & $48(16.0 \%)$ & $66(6.6 \%)$ & $2.70(1.81 \text { to } 4.01)^{\star *}$ & $3.48(2.21 \text { to } 5.48)^{\star *}$ \\
\hline Apgar score at $1<7(\%)$ & $3(1.0 \%)$ & $6(0.6 \%)$ & $1.68(0.42$ to 6.74$)$ & 2.47 (0.55 to 11.14$)$ \\
\hline Apgar score at $5<7(\%)$ & $0(0.0 \%)$ & $2(0.2 \%)$ & NA & NA \\
\hline Birth weight $(g) \pm S D$ & $2763.7 \pm 588.7$ & $3211.3 \pm 592.8$ & NA & NA \\
\hline Gestational days $(\mathrm{d}) \pm \mathrm{SD}$ & $259.3 \pm 16.2$ & $269.7 \pm 15.3$ & NA & NA \\
\hline
\end{tabular}

${ }^{*} \mathrm{P}<0.05$.

${ }^{* *} \mathrm{P}<0.01$.

†Adjusted ORs were calculated using logistic regression analyses, which were adjusted for maternal age, region, nulliparity, history of spontaneous abortion, history of diabetes and history of hypertension.

IUGR, intrauterine growth restriction; NA, not applicable; NICU, neonatal intensive care unit; SGA, small for gestational age; SLE, systemic lupus erythematosus.

was also associated with adverse fetal outcomes, including a high fetal loss risk, such as spontaneous abortion, particularly $\geq 10$ weeks, therapeutic abortion and stillbirth; higher rates of preterm births, caesarean sections, mainly elective and NICU admissions; and a significantly higher number of infants with growth restriction (eg, IUGR or SGA). The elevated risk conferred by SLE on each of these factors indicates that preconception assessments and antenatal monitoring are both important in pregnant women with SLE.

\section{Comparisons with previous studies}

We observed differences in several baseline demographic features between the women with SLE and those without in our cohort. Women with SLE were more likely to reside in a city and to have a nulliparous pregnancy than women in the non-SLE group. Importantly, women with SLE more frequently experienced a spontaneous abortion, which may potentially be associated with the high rate of adverse pregnancy outcomes observed in women with SLE. ${ }^{7}$ Differences in the prepregnancy incidences of chronic diseases were not observed between the women with and without SLE. This finding was also reported in two previous cohort studies. ${ }^{24} 25$

SLE is an important risk factor for fetal loss, including spontaneous abortion, therapeutic abortion and stillbirth. This finding was also reported in several previous cohort studies performed in other settings. ${ }^{424}{ }^{26-30}$ In the present study, the rates of stillbirth and spontaneous abortion in the SLE group were $1.8 \%$ and $3.3 \%$, respectively. These rates are much lower than the rates reported in other studies. This finding might be due to the use of different terms to define outcomes. However, in the present study, a higher rate of therapeutic abortion was observed in the SLE group $(6.2 \%$ in the SLE group vs $0.5 \%$ in the non-SLE group). Few previous studies have reported this outcome. ${ }^{72431}$ One systemic review reported the rate of elective abortion, but the OR was not significant (OR $1.19,95 \%$ CI 0.76 to 1.88$).{ }^{30}$ However, their definition of elective abortion differed from the definition used in the present study. In our study, therapeutic abortion was defined as an abortion performed for a therapeutic reason, but not elective abortions based on 
the mother's will. This definition may have contributed to the low rates of spontaneous abortions and stillbirths observed in our study, because pregnancies were terminated in some patients with severe cases of SLE using therapeutic abortion during an early trimester to maintain the health of the mother.

The results of our study confirm that PIH and pre-eclampsia are significant problems during pregnancy in women with SLE. In our study, nearly one-fifth of pregnant women with SLE experienced the complications of significant hypertension and pre-eclampsia, whereas only $5.8 \%-9.0 \%$ of the pregnancies in healthy women were affected by these conditions. The ORs remained significant, even after adjusting for confounding factors. SLE flares and pre-eclampsia are important and difficult to recognise in pregnant patients with SLE because both conditions are associated with proteinuria, deteriorating renal function, hypertension and sometimes even coexist. Although several guidelines or biomarkers, such as placental growth factor and vascular endothelial growth factor are available, they display limited utility in practical clinical settings. ${ }^{3}$ In our study, we considered several clinical manifestations together, including the onset time of hypertension, complement levels, antidsDNA levels, erythrocyte sedimentation rate, involvement of other organs and even responses to steroids. This differential diagnosis was a comprehensive process, and it was always assessed by an experienced gynaecologist or a rheumatologist in our study. Uncontrolled hypertension has been shown to predict poor pregnancy outcomes, ${ }^{3} 3132$ and guidelines recommend the implementation of measures to control blood pressure in these patients. ${ }^{3} 33$ In the present study, the rate of SLE flares was $24 \%$ and might have contributed to the adverse pregnancy outcomes, as reported previously. ${ }^{34}$ However, as the main goal of this article was to compare pregnancy outcomes between women with and without SLE, we did not further explore the effects of flares on pregnancy outcomes among the SLE group, which could be a future study direction.

Although a few cases of peripartum infections were reported in each group, the SLE group had a slightly higher proportion of infections. Clowse $e t a t^{b}$ and Nili $e t$ $a b^{55}$ reported similar results. This effect may be related to the nature of the immune dysregulation observed in patients with SLE or may be associated with the immunosuppressive nature of treatments for SLE.

Interestingly, we noticed a significantly lower rate of GDM in women with SLE than in the controls $(5.6 \%$ vs $11.5 \%$ ), even after adjusting for confounding factors. Only one previous study reported the same result. In the study by McGrory et al, none of the patients in the SLE group developed GDM, whereas $12 \%$ of patients in the non-SLE group developed GDM. ${ }^{31}$ Clowse $e t$ al reported a higher rate of pre-GDM in the SLE group, potentially because these patients were administered corticosteroids during pregnancy. ${ }^{6}$ Further studies of larger populations should be performed.
The rate of caesarean section surgeries was surprisingly high in our study (SLE vs non-SLE: $85 \%$ vs $55.6 \%$ ), and it was far higher than the rates reported in most previous studies. A national US population-based study performed in 2000-2003 reported a $36.6 \%$ caesarean rate in patients with SLE. ${ }^{6}$ Two studies conducted in Northern Europe reported caesarean rates of $32 \%-39 \%$ in pregnant women with SLE and 16\%-24\% in non-SLE pregnant women. ${ }^{5}{ }^{56}$ However, Saavedra et al reported a high caesarean rate (77.1\%-86.5\%) in a Latin American SLE group, consistent with our result. The main explanations were Mexican sociocultural, economic, medical-legal and biomedical factors. ${ }^{37}$ Although one study reported the opposite result (SLE vs non-SLE: $30 \%$ vs 53\%), the authors recruited patients who had received a kidney transplant, which may have contributed to the difference in their results. Although the absolute rate was high in both groups in our study, SLE was still a risk factor for caesarean section. Furthermore, most patients with SLE tended to undergo an elective caesarean, mainly because doctors and patients tended to choose caesarean section to prevent complications related to SLE during delivery. The extremely high caesarean section rate observed in our study reflects the true clinical situation in China. In 2008, WHO suggested that nearly half of all births in China are delivered via caesarean section. ${ }^{38}$ Some of the potential reasons for this high rate include a fear of pain and accidents during vaginal birth, an uneasy doctor-patient relationship, the profitability of caesarean sections for the hospital, and increases in the number of babies with macrosomia at birth and the number of pregnancies in older women. ${ }^{39}$

In our study, the percentages of neonates who were diagnosed with IUGR or SGA and mean birth weights were significantly higher in the SLE group than in the control group. Both of these terms (IUGR/SGA) are used as indicators of fetal growth restriction, but each involves a different mechanism. The definitions, we used for these terms, are provided in the methods section. Our findings are consistent with previous studies reporting growth restriction rates of 10\%-28\% in SLE groups. ${ }^{572540}$ The risk of neonatal death is threefold higher in growth-restricted neonates than in neonates with a normal weight. ${ }^{41}$ These infants also have high risk of developing other severe conditions, such as cardiovascular disease, infection and neurodevelopmental retardation. ${ }^{42}$ The risk of preterm birth was also threefold higher in both the very preterm or moderate to late preterm subgroups of women with SLE in our study, consistent with previous studies. ${ }^{45} 724364043$ Moreover, patients with SLE had a high risk of PPROM, but not PROM, which has not been reported in previous studies. The explanation for this finding might be that immune complexes cause vascular inflammation that contributes to a hypercoagulable state and subsequently reduces placental and umbilical artery blood flow and decreases placental perfusion and villus structure, thereby affecting fetal growth and prematurity. ${ }^{1844}$ Not surprisingly, the higher rates of prematurity and growth restriction observed in the SLE group led to a 
higher NICU admission rate. However, differences in the Apgar scores of the neonates were not observed at either 1 or $5 \mathrm{~min}$, and the absolute incidence of moderate or severe hypoxia was very low in both group. This finding contrasts the result described in the study Wallenius et $a l,{ }^{36}$ but this discrepancy may be because the size of the SLE group was small in the present study.

Four live births with congenital malformations occurred in the SLE group and 10 occurred in the control group, but the difference between the two groups was not significant. Among the four infants with congenital malformations in the SLE group, two had renal problems and one had polydactyly. Additionally, three cases of malformation in the SLE group were detected before 28 weeks and included one case of dextrocardia and two cases of maldevelopment of cardiac anatomy. These three cases were treated with a therapeutic abortion. Compared with previous studies, we reported a relatively low rate of congenital malformations in the present study. Wallenius et $a b^{6}$ and Liu $e t a l^{45}$ reported a rate of malformation of approximately $6 \%-7 \%$ in neonates born to women with SLE from approximately 2008-2009, and Rahman et al reported a rate of $2 \%$ from 1970 to $1995 .{ }^{46}$ One potential reason for these differences in the incidence of congenital malformations is the extensive improvements in the prenatal diagnosis and antenatal management of these patients.

As medication is an important contributor to the pregnancy outcomes in women with SLE, we also collected the medication data in the present study. Steroids were the most common medication used by patients with SLE, but the exposure should be minimised during pregnancy. However, short-term administration of high doses should be used during a disease flare, and doses must be administered at the time of delivery. ${ }^{3}$ Immunosuppressive therapy for SLE is often used to treat a flare. Azathioprine is the most commonly used drug during pregnancy, because it has proven safe for use during pregnancy. ${ }^{47}$ Antimalarial agents are now a first-line therapy for SLE during pregnancy. They are safe for pregnant women, and are proven to be associated with a reduced risk of congenital heart block in neonates. ${ }^{48}$ Aspirin has been proven to prevent pre-eclampsia in women with SLE. ${ }^{49}$ LMWH is safe and easy to administer, and along with low-dose aspirin, LMWH is routinely used in obstetric patients with APS. ${ }^{50}$

\section{Strengths and limitations}

The present study is noteworthy for several reasons. Pregnancy has been reported to be risky for women with SLE, due to the high rates of caesarean deliveries, infections, gestational hypertension and pre-eclampsia. However, this study is the first to compare maternal and fetal outcomes between pregnant women with and without SLE in a Chinese cohort. A nationwide population-based cohort was evaluated in Taiwan in 2010, but that study focused on only three fetal outcomes. ${ }^{25}$ Additionally, the present study included large samples of both patients with SLE and controls, comprising a total of 1350 individuals, and no data were missing for any of these patients. Furthermore, the maternal and fetal outcomes evaluated in this study were comprehensive and reflect almost every key aspect of pregnancy.

Nevertheless, our study has some limitations. First, as a retrospective study, this study has inherent biases, including selection bias and information bias. Second, the clinical records lacked some details regarding baseline population characteristics, including the level of education, body mass index, daily activity and family income, of some of the included participants. These factors may be confounding factors in the present study. Finally, data for some variables, such as 24-hour urinary protein, complement, auto-antibody and aPL antibody levels, were not available for the non-SLE group because we do not perform these laboratory tests on patients without SLE manifestations. Therefore, we were unable to compare these variables between the two groups.

The generalisability of this study should be interpreted with caution due to this study conducted in a single tertiary university hospital in China.

\section{CONCLUSIONS}

In conclusion, the risks of gestational hypertension, pre-eclampsia, peripartum infection, PPROM and elective caesarean birth are higher and the risk of GDM is lower in pregnant women with SLE than in women without SLE. Additionally, SLE contributes to higher rates of fetal loss, prematurity, fetal growth restriction and NICU admission. We, therefore, suggest that women with SLE should have full access to preconception counselling, choose an optimal time for pregnancy and undergo close monitoring during antenatal care by both rheumatologists and obstetricians. Future large cohort studies should focus on antenatal management, including laboratory assessments and medication use, in pregnant women with SLE.

Contributors JW, JM, CB, W-HZ and WD were each responsible for the conception and design of the study. JW, JM and WD had full access to all of the data in the study. JW and JM collected the data. JW and W-HZ analysed and interpreted the data. JW and JM drafted the initial manuscript. W-HZ, CB and WD revised the manuscript. All authors read and approved the final version of the manuscript.

Funding The work was supported by funding from the Shanghai Municipal Health and Family Planning Commission (15GWZK0701).

\section{Competing interests None declared.}

Patient consent Detail has been removed from this case description/these case descriptions to ensure anonymity. The editors and reviewers have seen the detailed information available and are satisfied that the information backs up the case the authors are making.

Ethics approval The research protocol used in this study was reviewed and approved by the Ethics Committee of Ren Ji Hospital, Shanghai Jiao Tong University School of Medicine. [2017-113]

Provenance and peer review Not commissioned; externally peer reviewed. Data sharing statement № additional data are available.

Open Access This is an Open Access article distributed in accordance with the Creative Commons Attribution Non Commercial (CC BY-NC 4.0) license, which permits others to distribute, remix, adapt, build upon this work non-commercially, and license their derivative works on different terms, provided the original work is 
properly cited and the use is non-commercial. See: http://creativecommons.org/ licenses/by-nc/4.0/

(c) Article author(s) (or their employer(s) unless otherwise stated in the text of the article) 2018. All rights reserved. No commercial use is permitted unless otherwise expressly granted.

\section{REFERENCES}

1. Carter EE, Barr SG, Clarke AE. The global burden of SLE: prevalence, health disparities and socioeconomic impact. Nat Rev Rheumatol 2016:12:605-20.

2. Meyer O. Making pregnancy safer for patients with lupus. Joint Bone Spine 2004;71:178-82.

3. Lateef A, Petri M. Systemic Lupus Erythematosus and Pregnancy. Rheum Dis Clin North Am 2017;43:215-26.

4. Molokhia M, Maconochie N, Patrick AL, et al. Cross-sectional analysis of adverse outcomes in 1,029 pregnancies of AfroCaribbean women in Trinidad with and without systemic lupus erythematosus. Arthritis Res Ther 2007;9:R124.

5. Jakobsen IM, Helmig RB, Stengaard-Pedersen K. Maternal and foetal outcomes in pregnant systemic lupus erythematosus patients: an incident cohort from a stable referral population followed during 1990-2010. Scand J Rheumatol 2015;44:85.

6. Clowse ME, Jamison M, Myers E, et al. A national study of the complications of lupus in pregnancy. Am J Obstet Gynecol 2008;199:127.e1-127.e6. e121-126.

7. Barnado A, Wheless L, Meyer AK, et al. Pregnancy outcomes among African-American patients with systemic lupus erythematosus compared with controls. Lupus Sci Med 2014;1:e000020.

8. Hochberg MC. Updating the American College of Rheumatology revised criteria for the classification of systemic lupus erythematosus. Arthritis Rheum 1997;40:1725.

9. Wilson WA, Gharavi AE, Koike T, et al. International consensus statement on preliminary classification criteria for definite antiphospholipid syndrome: report of an international workshop. Arthritis Rheum 1999;42:1309-11.

10. Danyliv A, Gillespie P, O'Neill C, et al. Short- and long-term effects of gestational diabetes mellitus on healthcare cost: a cross-sectional comparative study in the ATLANTIC DIP cohort. Diabet Med 2015;32:467-76.

11. Tunçalp O, Souza JP, Gülmezoglu M. World Health Organization. New WHO recommendations on prevention and treatment of postpartum hemorrhage. Int J Gynaecol Obstet 2013;123:254-6.

12. WHO. World health organization recommendations for prevention and treatment of maternal peripartum infections. Geneva: World Health Organization, 2015

13. Say L, Chou D, Gemmill A, et al. Global causes of maternal death: a WHO systematic analysis. Lancet Glob Health 2014;2:e323-33.

14. Ruperto N, Hanrahan LM, Alarcón GS, et al. International consensus for a definition of disease flare in lupus. Lupus 2011;20:453-62.

15. Clowse ME, Magder LS, Petri M. The clinical utility of measuring complement and anti-dsDNA antibodies during pregnancy in patients with systemic lupus erythematosus. J Rheumatol 2011;38:1012-6.

16. Xie X, Gou W, Di W, et al. Obstetrics and gynecology people's medical publishing house. 8th edn, 2013:47-50.

17. Tavares Da Silva F, Gonik B, McMillan M, et al. Stillbirth: Case definition and guidelines for data collection, analysis, and presentation of maternal immunization safety data. Vaccine 2016;34:6057-68.

18. Valero de Bernabé J, Soriano T, Albaladejo R, et al. Risk factors for low birth weight: a review. Eur J Obstet Gynecol Reprod Biol 2004;116:3-15

19. Goto E. Maternal anthropometry to predict small for gestational age: a meta-analysis. Eur J Obstet Gynecol Reprod Biol 2016;203:193-8.

20. Jehan I, Harris H, Salat S, et al. Neonatal mortality, risk factors and causes: a prospective population-based cohort study in urban Pakistan. Bull World Health Organ 2009;87:130-8.

21. WHO. Preterm birth. World health organization fact sheet, 2017.

22. Gagnon AJ, Merry L, Haase K. Predictors of emergency cesarean delivery among international migrant women in Canada. Int $\mathrm{J}$ Gynaecol Obstet 2013;121:270-4.

23. Iliodromiti S, Mackay DF, Smith GC, et al. Apgar score and the risk of cause-specific infant mortality: a population-based cohort study. Lancet 2014;384:1749-55.

24. Yan Yuen S, Krizova A, Ouimet JM, et al. Pregnancy outcome in systemic lupus erythematosus (SLE) is improving: Results from a case control study and literature review. Open Rheumatol $J$ 2008;2:89-98.
25. Chen $\mathrm{CY}$, Chen $\mathrm{YH}$, Lin HC, et al. Increased risk of adverse pregnancy outcomes for hospitalisation of women with lupus during pregnancy: a nationwide population-based study. Clin Exp Rheumatol 2010;28:49-55.

26. Zhan Z, Yang Y, Zhan Y, et al. Fetal outcomes and associated factors of adverse outcomes of pregnancy in southern Chinese women with systemic lupus erythematosus. PLoS One 2017;12:e0176457.

27. Lateef $A$, Petri M. Managing lupus patients during pregnancy. Bailliere's best practice and research in clinical rheumatology. 2013:27, 435-47.

28. Smyth A, Oliveira GH, Lahr BD, et al. A systematic review and meta-analysis of pregnancy outcomes in patients with systemic lupus erythematosus and lupus nephritis. Clin J Am Soc Nephrol 2010;5:2060-8.

29. Wei Q, Ouyang Y, Zeng W, et al. Pregnancy complicating systemic lupus erythematosus: a series of 86 cases. Arch Gynecol Obstet 2011;284:1067-71.

30. Bundhun PK, Soogund MZ, Huang F. Impact of systemic lupus erythematosus on maternal and fetal outcomes following pregnancy: A meta-analysis of studies published between years 2001-2016. J Autoimmun 2017;79:17-27.

31. McGrory CH, McCloskey LJ, DeHoratius RJ, et al. Pregnancy outcomes in female renal recipients: a comparison of systemic lupus erythematosus with other diagnoses. Am J Transplant 2003;3:35-42.

32. Imbasciati E, Tincani A, Gregorini G, et al. Pregnancy in women with pre-existing lupus nephritis: predictors of fetal and maternal outcome. Nephrol Dial Transplant 2009;24:519-25.

33. Gladman DD, Tandon A, Ibañez D, et al. The effect of lupus nephritis on pregnancy outcome and fetal and maternal complications. $J$ Rheumatol 2010;37:754-8.

34. Saavedra MA, Sánchez A, Morales S, et al. Primigravida is associated with flare in women with systemic lupus erythematosus. Lupus 2015;24:180-5

35. Nili F, McLeod L, O'Connell C, et al. Maternal and neonatal outcomes in pregnancies complicated by systemic lupus erythematosus: a population-based study. J Obstet Gynaecol Can 2013;35:323-8.

36. Wallenius M, Salvesen KÅ, Daltveit AK, et al. Systemic lupus erythematosus and outcomes in first and subsequent births based on data from a national birth registry. Arthritis Care Res 2014;66:1718-24.

37. Saavedra MÁ, Miranda-Hernández D, Sánchez A, et al. Pregnancy outcomes in women with childhood-onset and adult-onset systemic lupus erythematosus: a comparative study. Rheumatol Int 2016;36:1431-7.

38. Lumbiganon P, Laopaiboon M, Gülmezoglu AM, et al. Method of delivery and pregnancy outcomes in Asia: the WHO global survey on maternal and perinatal health 2007-08. Lancet 2010;375:490-9.

39. Mi J, Liu F. Rate of caesarean section is alarming in China. Lancet 2014;383:1463-4.

40. Barnabe C, Faris PD, Quan H. Canadian pregnancy outcomes in rheumatoid arthritis and systemic lupus erythematosus. Int $J$ Rheumatol 2011;2011:1-6.

41. Garite TJ, Clark R, Thorp JA. Intrauterine growth restriction increases morbidity and mortality among premature neonates. Am J Obstet Gynecol 2004;191:481-7.

42. Regal JF, Gilbert JS, Burwick RM. The complement system and adverse pregnancy outcomes. Mol Immunol 2015;67:56-70.

43. Dhar JP, Essenmacher LM, Ager JW, et al. Pregnancy outcomes before and after a diagnosis of systemic lupus erythematosus. Am J Obstet Gynecol 2005:193:1444-55.

44. Stone S, Pijnenborg R, Vercruysse L, et al. The placental bed in pregnancies complicated by primary antiphospholipid syndrome. Placenta 2006;27:457-67.

45. Liu J, Zhao Y, Song Y, et al. Pregnancy in women with systemic lupus erythematosus: a retrospective study of 111 pregnancies in Chinese women. J Matern Fetal Neonatal Med 2012;25:261-6.

46. Rahman P, Gladman DD, Urowitz MB. Clinical predictors of fetal outcome in systemic lupus erythematosus. $J$ Rheumatol 1998;25:1526-30.

47. Saavedra MÁ, Sánchez A, Morales S, et al. Azathioprine during pregnancy in systemic lupus erythematosus patients is not associated with poor fetal outcome. Clin Rheumatol 2015;34:1211-6.

48. Izmirly PM, Costedoat-Chalumeau N, Pisoni CN, et al. Maternal use of hydroxychloroquine is associated with a reduced risk of recurrent anti-SSA/Ro-antibody-associated cardiac manifestations of neonatal lupus. Circulation 2012:126:76-82.

49. Schramm AM, Clowse ME. Aspirin for prevention of preeclampsia in lupus pregnancy. Autoimmune Dis 2014;2014:1-8.

50. Schreiber K, Sciascia S, de Groot PG, et al. Antiphospholipid syndrome. Nat Rev Dis Primers 2018;4:17103. 